\title{
Incidence and Mortality Related to Gastrointestinal Bleeding, and the Effect of Tranexamic Acid on Gastrointestinal Bleeding
}

\author{
Ylva Scherdin ${ }^{\mathrm{a}}$, Ingvar Halldestam ${ }^{\mathrm{a}}$, Stefan Redeen ${ }^{\mathrm{a}, \mathrm{b}}$
}

\begin{abstract}
Background: Gastrointestinal bleeding is a common and potentially life-threatening condition. The incidence of gastrointestinal bleeding has not decreased despite new prophylaxis and treatments. Ulcer is still one of the most common etiologies for upper gastrointestinal bleeding. It is routinely treated with proton pump inhibitors (PPIs) and endoscopic interventions, sometimes endovascular procedures, and rarely today, open surgery with suture to stop the bleeding. The fibrinolytic tranexamic acid (TXA) has a role in bleeding treatment, and is routinely used for example within trauma care, postpartum bleeding and orthopedic surgery. The aim of this study is to assess the incidence of gastrointestinal bleeding. A further aim was to investigate if TXA has any role in medical treatment of gastrointestinal bleeding today.
\end{abstract}

Methods: We performed a retrospective cohort study with a review of medical records, involving patients with clinical signs of gastrointestinal bleeding and endoscopically verified ulcers between the years of 2010 and 2016 at the University Hospital of Linkoping, Sweden. The cities of Motala and Linkoping have the primary acute admissions at this Hospital.

Results: We found in total 1,331 patients with gastrointestinal bleeding. The overall incidence for patients with gastrointestinal bleeding was 98.6 (98.6/100,000 inhabitants and year). For those with endoscopically verified ulcer (386 patients), the incidence for peptic ulcer was 28.6/100,000/year. In the group with endoscopically verified ulcer, 25 patients died, giving the 30 -day mortality of $6.4 \%$. TXA is still used for treatment of bleeding ulcers. We had two groups, those with and without TXA treatment. They were equal in age, gender and comorbidity. Clinically we saw no major differences in respect to hemodynamic stability. There were more patients with overt bleeding symptoms in the TXA group. We also saw more patients in need of

Manuscript submitted March 30, 2021, accepted May 28, 2021

Published online June 19, 2021

aDepartment of Surgery and Department of Biomedical and Clinical Sciences, Linkoping University, Linkoping, Sweden

${ }^{b}$ Corresponding Author: Stefan Redeen, Department of Surgery and Department of Biomedical and Clinical Sciences, Linkoping University, Linkoping, Sweden. Email: stefan.redeen@regionostergotland.se

doi: https://doi.org/10.14740/gr1383 intensive care in the TXA group.

Conclusions: The incidence of gastrointestinal bleeding has not significantly decreased during the last years. There was no significant positive effect of TXA in patients with upper gastrointestinal bleeding in this study. The difference between the two groups is probably more a question of whom we treat with TXA (e.g., the patients in worse condition or at higher risk) than a difference in drug effect. It is time to quit with TXA treatment in all patients with gastrointestinal bleeding, even those at intensive care unit (ICU).

Keywords: Incidence; Gastrointestinal bleeding; Melena; Gastroscopy; Ulcer; Re-bleeding; Tranexamic acid; Mortality

\section{Introduction}

Patients with upper gastrointestinal bleeding (UGIB) are common at the emergency hospitals, almost all over the world. There are morbidity, mortality and even big costs for the society regarding these patients. Updated use of new anticoagulants, in different combinations, aimed to prevent further cardiovascular events, produce some of these bleeding occasions. The UGIB patients sometimes suffer from severe comorbidity, contributing to potential, acute life-threatening bleeding complications. The bleeding may be occult, presenting with fatigue, dizziness and other unspecific symptoms, or overt with hematemesis, melena or hematochezia. A correctly used scoring system often gives a good understanding on how to handle your patient [1-3]. Some patients could go home from the emergency department, and instead be offered a planned follow-up examination in a few days [3]. The majority have to stay at the hospital for acute care in some way, often gastroscopy within $24 \mathrm{~h}$. Bleedings at admissions are of unknown etiology and location. Some are from the upper part of the gastrointestinal tract, others from the lower part. UGIB, e.g., bleeding from proximal of ligament Treitz, accounts for the majority; and peptic ulcers in the stomach or duodenum are still the most common cause of UGIB, at least, what we have seen until today [3-5].

The overall incidence of gastrointestinal bleeding in a study from the UK was 103/100,000/year, increasing with age, where the mortality was found at $14 \%$ [6]. In another study 
from USA, the incidence was 61 - 78/100,000/year in general, and for ulcer 21/100,000/year [7]. In a European study, the incidence was $40-150 / 100,000 /$ year in general, and for duodenal and gastric ulcer 11 - 59/100,000/year [8]. In a study from USA from 2018, the incidence was decreasing from 81 to $67 / 100,000 /$ year [5]. Another study had an incidence for UGIB at $84-160 / 100,000$ and a mortality around 10\% [9]. Peptic ulcer incidence per se in a study from 2012 was decreasing from 48.7 to $32.1 / 100,000 /$ year [10].

The amount of morbidity and even mortality among patients with UGIB is high. Mortality related to UGIB is around $2-14 \%$, increasing with age [4, 6-8].

The currently recommended medical treatment for bleeding ulcer is proton pump inhibitors (PPIs) given with continuous infusion or intermittent injections, combined with endoscopic intervention with injection therapy including epinephrine and with at least one of contact thermal, mechanical therapy, or injection of some sclerosing agents [3, 7, 9]. Theoretically, another medical treatment, tranexamic acid (TXA) is appealing, but the evidence is debated [11].

It is shown that TXA administration intravenously can reduce maternal mortality from post-partum hemorrhage without increasing the risk for thromboembolic events [12]. It is also used within elective orthopedic, gynecology and urologic surgery, decreasing perioperative bleeding and the need for transfusions [13-15]. In the CRASH-2 study, a big multicenter study involving 20,211 adult trauma patients with or at risk for significant bleeding, also showed TXA's effectiveness in reducing mortality, if given early in the process, like other studies have shown [16-18].

Regarding UGIB, there are suggestions that the antifibrinolytic effect of TXA may decrease the need for acute endoscopy and convert it to daytime elective procedure with subsequent less risk for aspiration and the possibility of using more experienced personal [19]. However, TXA has not proved effect in reducing mortality rate and is not recommended routinely [20]. In 2011 TXA was removed from Swedish national guidelines, Swedish Agency for Health Technology Assessment and Assessment of Social Services (www.SBU. se). However, we can still see that many doctors use TXA in UGIB treatment. Perhaps some think there are uncertain findings to refrain from the use of TXA in gastrointestinal bleeding patients, especially when the patient is circulatory affected.

The aim of this study was to study the incidence and mortality of gastrointestinal bleeding among adults. A further aim was to investigate if TXA today have any role in medical treatment of gastrointestinal bleeding in a cohort from a Swedish University Hospital.

\section{Materials and Methods}

\section{Ethics}

This retrospective cohort study was performed during 2017 2019 in accordance with the ethical declaration from the updated Helsinki Declaration. Information about each patient was collected from medical records at the University Hospital of Linkoping in the county of Ostergotland, Sweden. The study was performed with approval from the local ethical committee.

\section{Study population}

We selected diagnoses involving gastrointestinal bleeding from International Statistical Classification of Diseases (ICD10), including UGIB, melena, hematemesis and hematochezia. We used assistance from our care administrator, daily using the ICD system. We gathered all patients that had been admitted at the acute surgical ward at the University Hospital in Linkoping, during the years of 2010 - 2016, given the diagnosis of gastrointestinal bleeding.

The University Hospital in Linkoping serves the cities of Motala and Linkoping, with 42,363 (inhabitants in Motala was 41,955 in 2010, gradually increasing to 43,250 at the year of 2016) and 149,053 (inhabitants in Linkoping was 146,422 in 2010, gradually increasing to 155,539 at the year of 2016) inhabitants, respectively during the studied years. Statistics come from the public statistics of Motala and Linkoping municipality.

The University Hospital of Linkoping is the only hospital for gastrointestinal bleedings from Motala and Linkoping, during the study period all patients were admitted to the surgical department.

A review of the medical records was done, where we found 1,331 patients with signs of gastrointestinal bleeding at arrival. Two patients without a valid social security number, 496 patients without an endoscopic examination (esophagogastroduodenoscopy (EGD)), and 132 patients with other causes than ulcer of UGIB, were excluded from the TXA part in this study (Fig. 1). Normal gastroscopy was found in 314 patients.

\section{Study protocol}

Information about the patients were collected and registered in a file and categorized with patient factors (gender, age, pulse, blood pressure (BP), hemoglobin level, hematemesis, hematochezia, melena and abdominal pain). Lastly, medical outcome measured in re-bleeding events, hospital stay (in days), days at the intensive care unit (ICU), 30-day mortality and units of blood needed were registered.

\section{Statistical analysis}

Incidence was calculated with number of patients with gastrointestinal bleeding related to inhabitants in Motala and Linkoping, regarding to population in each included year, in both Motala and Linkoping. Occurrence per 100,000/year was used. Mortality was calculated in the same manner.

Background variables were calculated with mean and median for each group. Patients treated with or without TXA formed two groups, and were further analyzed with multivariate analysis. Symptoms, previous medical problems and outcomes including ICU stay were calculated and expressed as percent in each group. Median value was calculated for the 


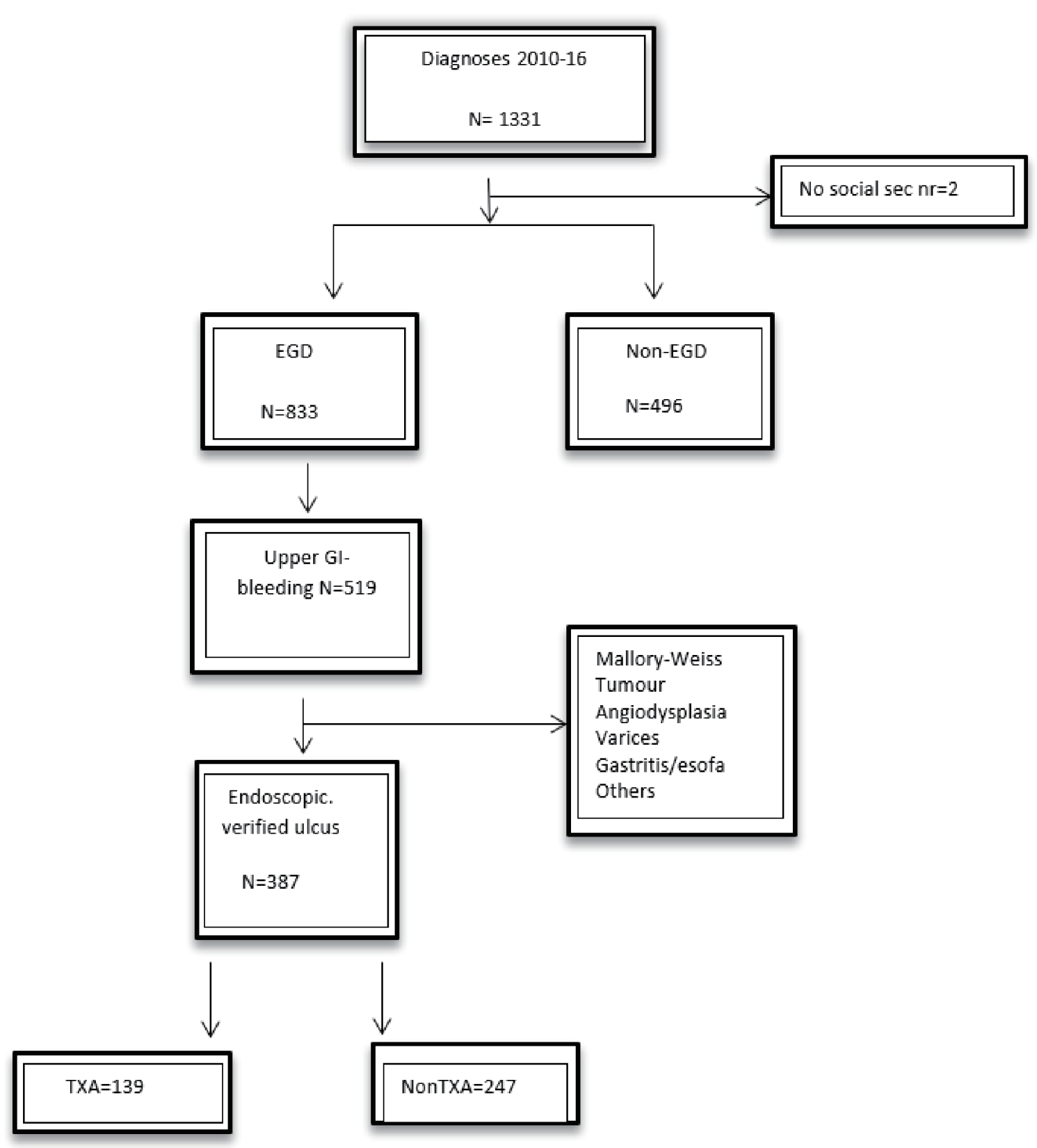

Figure 1. The study population. EGD: esophagogastroduodenoscopy; TXA: tranexamic acid; GI: gastrointestinal.

number of days admitted in the surgical ward. In every analysis $\mathrm{P}$ value $<0.05$ was regarded as significant.

\section{Results}

In total, we found 1,331 patients presented at the emergency ward with gastrointestinal bleeding during the study period. The overall incidence for patients with gastrointestinal bleeding was 98.6 (98.6/100,000 inhabitants and year). There were 386 patients with endoscopically verified ulcer, and the incidence for peptic ulcer was 28.6/100,000/year in this study.
Regarding 30-day mortality, 65 patients died in the general group with gastrointestinal bleeding. We had data from 905 patients, and there were data missing from 426 patients, giving $7.2 \%, 30$-day mortality. In the group with endoscopically verified ulcer, 25 patients died, giving 6.4\%, 30-day mortality. In this cohort $(\mathrm{n}=1,331)$, we had 101 patients with an age $\geq$ 90 years, 474 patients were $\geq 80$ years, 784 patients were $\geq 70$ years and 1,034 over the age of $\geq 60$ years.

There were in total 577 patients who were on anticoagulants. We had data from 1,078 patients and data missing from 253 patients. In total, 387 patients had cardiovascular disease, although there were no data about cardiovascular disease from 
Table 1. Physiologic Characteristics of Patients With Endoscopically Verified Ulcer Bleeding at Arrival to Hospital (Mean and Medium Values)

\begin{tabular}{llll} 
& Total & TXA group & Non-TXA group \\
\hline Female, n (\%) & $153(39.5)$ & $51(36.7)$ & $101(41.1)$ \\
Male, n (\%) & $234(60.5)$ & $88(63.3)$ & $146(58.9)$ \\
Mean age (range) & $71.2(14-97)$ & $72.3(23-97)$ & $70.6(14-93)$ \\
Mean pulse (range) & $87(43-140)$ & $89(47-143)$ & $85(43-140)$ \\
Mean BP (range) & $127(60-210)$ & $122(60-120)$ & $130(65-200)$ \\
Mean Hb (range) & $101.3(41-90)$ & $93.8(41-166)$ & $105.5(48-190)$ \\
Hematemesis, n (\%) & $123(31.7)$ & $58(42)$ & $65(26)$ \\
Hematochezia, n (\%) & $63(16.2)$ & $30(22)$ & $33(13)$ \\
Melena, n (\%) & $217(56)$ & $97(70)$ & $120(48)$ \\
Abdominal pain, n (\%) & $84(21.7)$ & $17(12)$ & $67(27)$ \\
\hline
\end{tabular}

TXA: tranexamic acid; BP: blood pressure.

253 patients, leaving $35.9 \%$ with cardiovascular disease.

In Table 1, we present the physiologic characteristics at arrival to the hospital. There were more male than female patients in both the TXA and the non-TXA group. The mean age did not differ between the groups. The median age was 72.3 and 70.6 years in the TXA group and the non-TXA group, respectively. BP, hemoglobin levels or clinical symptoms like hematemesis, hematochezia show little differences. Melena and abdominal pain were more common in the non-TXA group. There is some difference in the presentation of clinical symptoms in the two groups. There were more patients with more overt symptoms such as hematemesis, hematochezia and melena in the TXA group compared to patients that were not given TXA.

Regarding comorbidity and/or the use of anticoagulation treatment in the TXA and non-TXA group, 34.8\% of patients in the TXA group had a history of cardiac disease and $50.7 \%$ were treated with some kind of anticoagulation medication. This is compared with $36.2 \%$ with cardiac problems in the non-TXA group and $48.3 \%$ with anticoagulation treatment.

However, the clinical state in the matter of hemodynamic stability showed less difference regarding BP. The median BP was $120 \mathrm{~mm} \mathrm{Hg}$ in the TXA group and 128 in the other group. Median pulse was $85 / \mathrm{min}$ and $90 / \mathrm{min}$, respectively, almost no difference.
Table 2 shows patients' data presented from the medical outcome and specific observations during hospital stay. The occurrence of re-bleeding, 30-day mortality, cardiovascular disease and anticoagulants were more frequent in the nonTXA group.

In Table 3, the multivariate analysis is presented for 828 possible patients from the general group with gastrointestinal bleeding, with TXA as dependent variable. Pulse, hematemesis and hospital stay (days) are significant. All the other data like BP, ICU care, re-bleeding and units of blood are not significant. Except for hospital stay and units of blood, the physiologic parameter after treatment gives no significant outcome.

The multivariate analysis for the 378 endoscopically verified ulcer patients with dependent variable TXA is presented in Table 4. Only hematemesis and hematochezia give a significant value. All other parameters give no significant results in this study. No physiologic parameter after treatment gives any significant result.

\section{Discussion}

Gastrointestinal bleeding is a common and sometimes lifethreatening condition, fatal mainly due to cardiovascular comorbidity and an acute event developed by acute gastrointes-

Table 2. Medical Outcome and Specific Observations During Hospital Stay

\begin{tabular}{llll}
\hline Outcome & Total & TXA group & Non-TXA group \\
\hline Re-bleeding, n & 24 & $8(6 \%)$ & $16(6 \%)$ \\
Hospital stay (in days) & $4.7(0-37)$ & $4.3(0-27)$ & $4.9(0-37)$ \\
ICU, n & 40 & $24(17 \%)$ & $16(6 \%)$ \\
30-day mortality & 25 & $9(6 \%)$ & $17(7 \%)$ \\
Units of blood, n (\%) & 162 & $105(1-13,76 \%)$ & $57(1-16,23 \%)$ \\
Cardiovascular disease & 145 & $52(37 \%)$ & $93(38 \%)$ \\
Anticoagulants & 189 & $73(52 \%)$ & $116(47 \%)$ \\
\hline
\end{tabular}

TXA: tranexamic acid; ICU: intensive care unit. 
Table 3. Multivariate Analysis of All Endoscopically Examined (Dependent Variable: TXA, $N=828$ )

\begin{tabular}{llll}
\hline Independent variables & Coefficient & P value & 95\% confidence interval \\
\hline Sex & 0.059 & 0.077 & $-0.006,0.125$ \\
Age & 0.002 & 0.025 & $0.000,0.004$ \\
Pulse & 0.002 & 0.002 & $0.000,0.004$ \\
BP & 0.000 & 0.277 & $-0.001,0.000$ \\
Hematemesis & 0.115 & 0.001 & $0.044,0.186$ \\
ICU care & 0.022 & 0.253 & $-0.015,0.060$ \\
Hospital stay (in days) & -0.009 & 0.003 & $-0.016,-0.003$ \\
Re-bleeding & 0.006 & 0.933 & $-0.147,0.160$ \\
Units of blood & 0.030 & 0.000 & $0.015,0.045$ \\
\hline
\end{tabular}

TXA: tranexamic acid; BP: blood pressure; ICU: intensive care unit.

tinal bleeding. The mortality is increasing with age [6]. It is crucial that every patient is given optimal treatment, just in time. The knowledge and professional treatment is very important, because UGIB affects many people around the globe.

The treatment of UGIB is done primarily with endoscopy. Medical therapy could be important too, parallel to other therapies. This study gives some updated information about the incidence of gastrointestinal bleeding and useful information about TXA. Maybe it is time to stop the TXA treatment now, for all patients, even those with unstable circulation at the emergency wards and ICU. There are many important questions in this group. Regarding ethics, could we give a treatment that has unsure evidence about today? We do not think so. Even costs are a question, as the resources could be used in a better way.

The aim of this study was to study the incidence of gastro- intestinal bleeding and provide an overview of the use of TXA in patients with gastrointestinal bleeding due to ulcers and to compare groups to evaluate the effect of TXA treatment in a cohort.

Previously incidence studies give an incidence with a range of $40-150 / 100,000 /$ year [5-10]. That range is quite wide. Our study gives an overall incidence at 98.6/100,000/ year. There have not been big changes in the last years, as what we can see [10].

The population gets older in the western world today, often surviving other diseases such as cancer and cardiovascular events, which explains the occurrence of many patients with comorbidity and high age [21].

The gastric mucosa changes get more sensitive over time, and more frequently atrophic gastritis has been seen in higher age group [22-25]. All together gives a sensitive situation for

Table 4. Multivariate Analysis, Ulcer Patients (Dependent Variable: TXA, $N=378$ )

\begin{tabular}{llll}
\hline Independent variable & Coefficient & P value & 95\% confidence interval \\
\hline Sex & 0.009 & 0.840 & $-0.0838,0.103$ \\
Age & 0.001 & 0.259 & $-0.0013,0.004$ \\
Pulse & 0.001 & 0.283 & $-0.0010,0.003$ \\
BP & -0.001 & 0.248 & $-0.0029,0.000$ \\
Hematemesis & 0.205 & 0.000 & $0.1017,0.309$ \\
Hematochezia & 0.211 & 0.001 & $0.0853,0.337$ \\
Melena & 0.189 & 0.000 & $0.0873,0.290$ \\
Abdominal pain & -0.024 & 0.681 & $-0.139,0.091$ \\
Hb & 0.000 & 0.750 & $-0.002,0.001$ \\
Cardiovascular disease & -0.060 & 0.218 & $-0.157,0.036$ \\
PPI & 0.286 & 0.000 & $0.185,0.388$ \\
Units of blood & 0.010 & 0.383 & $-0.013,0.035$ \\
Re-bleeding & -0.122 & 0.204 & $-0.311,0.066$ \\
Hospital stay days & -0.005 & 0.306 & $-0.014,0.004$ \\
ICU care & 0.135 & 0.091 & $-0.021,0.292$ \\
30-day mortality & -0.055 & 0.552 & $-0.239,0.128$ \\
\hline
\end{tabular}

TXA: tranexamic acid; BP: blood pressure; PPI: proton pump inhibitor; Hb: hemoglobin; ICU: intensive care unit. 
the UGIB patients in an acute phase.

Regarding ulcer patients and incidence studies, we cannot find so many. We had an incidence of 28.6/100,000/year, and previous studies showed an incidence at 32.1 [10]. These findings are surprising. The background with Helicobacter pylori (H. pylori) eradication has been done for many years now, prophylaxis is recommended when there is indication. One question we cannot give an answer to is why it seems to be the same incidence today as the last $10-20$ years. Prophylaxis with anticoagulants is important for patients with cardiovascular diseases. The question is how many gastrointestinal bleeding occasions the anticoagulants produce; and if patients are given correct bleeding prophylaxis when they need prophylaxis [26-28]. That answer is not for this study. The 30-day mortality of $6.4 \%$ is in line with previous studies [9].

TXA is still used in clinical practice, although not routinely. It is maybe most used in situations with gastrointestinal bleeding and unstable circulation. This is in line with previous studies. TXA does not seem to have a significant, if any, positive effect on patients with gastrointestinal bleeding.

On an overview, the two groups with and without TXA are fairly equal regarding age and comorbidity. Nor does clinical presentation in means of hemodynamically stability differ; however patients given TXA had more overt bleeding symptoms such as hematemesis, which might be the answer to which patient group is given the treatment when it is no longer given routinely. Patients in a worse condition or at higher risk, e.g., with an ongoing bleeding, are given all medical support available including TXA for stabilization. More patients in the TXA group were treated in the ICU. Since the time of administration of TXA is not being considered, and all patients taken to the ICU were taken directly from the emergency room, the connection is not interpretable but probably also an effect of the tendency to treat more weak and ill patients with TXA. The most fragile and old patients are not evaluated in this study, since they were not undergoing EGD, and hence were excluded. The number of patients in this study could be a strength. A weakness could maybe be the problem with the definition of patients, in what specific occasion all medications are given.

Wuerth et al found that the hospitalization rate of UGIB in the USA decreased significantly by $21 \%$ from 2002 to 2012 , from 81 to 67 cases per 100,000 . We could not find in our material the same decrease in hospitalization, when using scoring instrument maybe another hospitalization number will be found. The incidence in general for UGIB and for ulcer bleeding was not changed, which is in line with our findings [5]. In another study from USA Laine et al found ulcer incidence at the same levels as this study [10].

The international multicenter randomized placebo-controlled trial (HALT-IT) with 164 hospitals in 15 countries including 12,009 patients concluded that TXA did not reduce death from gastrointestinal bleeding. Further, they conclude that TXA for the treatment of gastrointestinal bleeding outside the context of a randomized trial should not be used [29]. This is in line with our results from a randomized study.

In a review study with data from eight randomized trials on TXA from 2014 Bennett et al found that TXA could have some effect on mortality [30]. The evidences are not always clear.

In this study we cannot find any data that support treatment of bleeding ulcers with TXA, even those at ICU. This is new information. We have found that bleeding ulcer treatment could have updated treatment without TXA. We can stop use of TXA when we treat bleeding ulcers.

There might be limitations in this study. Some other patients were perhaps never hospitalized and therefore not included. Some patients were excluded because no gastroscopy was performed. Further limitations could be that we have no data about malignancies and thromboembolism during treatment with TXA. Some patients on anticoagulants could also affect the outcome. Some data are missing. We do not present any data from endoscopic or surgical complications.

This study is a single center cohort study. A multicenter randomized study could possibly give another perspective and outcome. The outcome could also be different if patients with other reasons than bleeding ulcers were included, some patients without hospital stay, another sample size, prior research and access to data could give other data.

The strength of this study is the sample size, valid data, all included patients have had a gastroscopy, prior research about TXA, access to data and that only one person collected data.

In conclusion, we found an incidence regarding gastrointestinal bleeding at the same level as previous studies both in general and specific for patients with ulcer disease. No big changes have taken place during the last 10 years, at least. The 30 -day mortality of ulcer patients was $6.4 \%$. Regarding TXA, we cannot see any significant difference when using TXA in this study. We do not need TXA for treatment for patients with gastrointestinal bleeding in the future.

\section{Acknowledgments}

The authors thank Helen Eriksson for data collection.

\section{Financial Disclosure}

None to declare.

\section{Conflict of Interest}

None to declare.

\section{Informed Consent}

The informed consent was not requested by the ethics committee.

\section{Author Contributions}

Ylva Scherdin: data collection, processing, calculation and writing. Ingvar Halldestam: planning, processing, calculation 
and writing, Stefan Redeen: idea and planning, data collection, processing, calculation, writing and final approval from all authors.

\section{Data Availability}

The data supporting the findings of this study are available from the corresponding author upon reasonable request.

\section{References}

1. Blatchford O, Murray WR, Blatchford M. A risk score to predict need for treatment for upper-gastrointestinal haemorrhage. Lancet. 2000;356(9238):1318-1321.

2. Rockall TA, Logan RF, Devlin HB, Northfield TC. Risk assessment after acute upper gastrointestinal haemorrhage. Gut. 1996;38(3):316-321.

3. Sung JJ, Chiu PW, Chan FKL, Lau JY, Goh KL, Ho LH, Jung HY, et al. Asia-Pacific working group consensus on non-variceal upper gastrointestinal bleeding: an update 2018. Gut. 2018;67(10):1757-1768.

4. Kim JS, Kim BW, Kim DH, Park CH, Lee H, Joo MK, Jung DH, et al. Guidelines for Nonvariceal Upper Gastrointestinal Bleeding. Gut Liver. 2020;14(5):560-570.

5. Wuerth BA, Rockey DC. Changing Epidemiology of Upper Gastrointestinal Hemorrhage in the Last Decade: A Nationwide Analysis. Dig Dis Sci. 2018;63(5):12861293.

6. Rockall TA, Logan RF, Devlin HB, Northfield TC. Incidence of and mortality from acute upper gastrointestinal haemorrhage in the United Kingdom. Steering Committee and members of the National Audit of Acute Upper Gastrointestinal Haemorrhage. BMJ. 1995;311(6999):222226.

7. Stanley AJ, Laine L. Management of acute upper gastrointestinal bleeding. BMJ. 2019;364:1536.

8. Gralnek IM, Dumonceau JM, Kuipers EJ, Lanas A, Sanders DS, Kurien M, Rotondano G, et al. Diagnosis and management of nonvariceal upper gastrointestinal hemorrhage: European Society of Gastrointestinal Endoscopy (ESGE) Guideline. Endoscopy. 2015;47(10):a1-46.

9. Kurien M, Lobo AJ. Acute upper gastrointestinal bleeding. Clin Med (Lond). 2015;15(5):481-485.

10. Laine L, Yang H, Chang SC, Datto C. Trends for incidence of hospitalization and death due to GI complications in the United States from 2001 to 2009. Am J Gastroenterol. 2012;107(8):1190-1195; quiz 1196.

11. Brenner A, Afolabi A, Ahmad SM, Arribas M, Chaudhri R, Coats T, Cuzick J, et al. Tranexamic acid for acute gastrointestinal bleeding (the HALT-IT trial): statistical analysis plan for an international, randomised, double-blind, placebo-controlled trial. Trials. 2019;20(1):467.

12. Shakur H, Beaumont D, Pavord S, Gayet-Ageron A, Ker $\mathrm{K}$, Mousa HA. Antifibrinolytic drugs for treating primary postpartum hemorrhage. Emergencias. 2020;32(3):203205.

13. Mina SH, Garcia-Perdomo HA. Effectiveness of tranexamic acid for decreasing bleeding in prostate surgery: a systematic review and meta-analysis. Cent European J Urol. 2018;71(1):72-77.

14. Pabinger I, Fries D, Schochl H, Streif W, Toller W. Tranexamic acid for treatment and prophylaxis of bleeding and hyperfibrinolysis. Wien Klin Wochenschr. 2017;129(9-10):303-316.

15. Klebanoff JS, Marfori CQ, Ingraham $\mathrm{CF}$, Wu CZ, Moawad GN. Applications of Tranexamic acid in benign gynecology. Curr Opin Obstet Gynecol. 2019;31(4):235239.

16. collaborators C-t, Shakur H, Roberts I, Bautista R, Caballero J, Coats T, Dewan Y, et al. Effects of tranexamic acid on death, vascular occlusive events, and blood transfusion in trauma patients with significant haemorrhage (CRASH-2): a randomised, placebo-controlled trial. Lancet. 2010;376(9734):23-32.

17. collaborators C-t. Effects of tranexamic acid on death, disability, vascular occlusive events and other morbidities in patients with acute traumatic brain injury (CRASH-3): a randomised, placebo-controlled trial. Lancet. 2019;394(10210):1713-1723.

18. El-Menyar A, Sathian B, Wahlen BM, Abdelrahman H, Peralta R, Al-Thani H, Rizoli S. Prehospital administration of tranexamic acid in trauma patients: A 1:1 matched comparative study from a level 1 trauma center. Am J Emerg Med. 2020;38(2):266-271.

19. Tavakoli N, Mokhtare M, Agah S, Azizi A, Masoodi M, Amiri H, Sheikhvatan M, et al. Comparison of the efficacy of intravenous tranexamic acid with and without topical administration versus placebo in urgent endoscopy rate for acute gastrointestinal bleeding: A double-blind randomized controlled trial. United European Gastroenterol J. 2018;6(1):46-54.

20. Atikah N, Singh G, Maulahela H, Cahyanur R. Tranexamic acid in the management of upper gastrointestinal bleeding: an evidence-based case report. Acta Med Indones. 2015;47(2):172-175.

21. Eckerblad J, Waldreus N, Stark AJ, Jacobsson LR. Symptom management strategies used by older community-dwelling people with multimorbidity and a high symptom burden - a qualitative study. BMC Geriatr. 2020;20(1):210.

22. Borch K, Jonsson KA, Petersson F, Redeen S, Mardh S, Franzen LE. Prevalence of gastroduodenitis and Helicobacter pylori infection in a general population sample: relations to symptomatology and life-style. Dig Dis Sci. 2000;45(7):1322-1329.

23. Sipponen P, Kimura K. Intestinal metaplasia, atrophic gastritis and stomach cancer: trends over time. Eur J Gastroenterol Hepatol. 1994;6(Suppl 1):S79-83.

24. Sipponen P, Maaroos HI. Chronic gastritis. Scand J Gastroenterol. 2015;50(6):657-667.

25. Redeen S, Petersson F, Kechagias S, Mardh E, Borch K. Natural history of chronic gastritis in a population-based cohort. Scand J Gastroenterol. 2010;45(5):540-549.

26. Burr N, Lummis K, Sood R, Kane JS, Corp A, Subramanian V. Risk of gastrointestinal bleeding with direct oral anticoagulants: a systematic review and network meta- 
analysis. Lancet Gastroenterol Hepatol. 2017;2(2):85-93.

27. Cheung KS, Leung WK. Gastrointestinal bleeding in patients on novel oral anticoagulants: Risk, prevention and management. World J Gastroenterol. 2017;23(11):19541963.

28. Ray WA, Chung CP, Murray KT, Smalley WE, Daugherty JR, Dupont WD, Stein CM. Association of Oral Anticoagulants and Proton Pump Inhibitor Cotherapy With Hospitalization for Upper Gastrointestinal Tract Bleeding.
JAMA. 2018;320(21):2221-2230.

29. Collaborators H-IT. Effects of a high-dose 24-h infusion of tranexamic acid on death and thromboembolic events in patients with acute gastrointestinal bleeding (HALTIT): an international randomised, double-blind, placebocontrolled trial. Lancet. 2020;395(10241):1927-1936.

30. Bennett C, Klingenberg SL, Langholz E, Gluud LL. Tranexamic acid for upper gastrointestinal bleeding. Cochrane Database Syst Rev. 2014;11:CD006640. 